\title{
LA VALIDEZ DE LOS PROCEDIMIENTOS DE REFORMA CONSTITUCIONAL FAST TRACK*
}

\author{
VALIDITY OF THE FAST TRACK \\ CONSTITUTIONAL AMENDMENTS
}

Fabio Enrique Pulido-Ortiz**

Fecha de recepción: 1 de marzo de 2017

Fecha de aceptación: 20 de abril de 2017

Disponibilidad en linea: 30 de junio de 2017

\section{Para Citar Este Artículo / To Cite This Article}

Pulido-Ortiz, Fabio Enrique, La validez de los procedimientos de reforma constitucional fast track, 30 International Law, Revista Colombiana de Derecho Internacional, 151-184 (2017). https://doi.org/10.11144/ Javeriana. il 15-30.vprc

doi:10.11144/Javeriana.i115-30.vprc

* Artículo de investigación que forma parte del Proyecto DER-49-2016 apoyado por la Dirección de investigaciones de la Universidad de La Sabana.

** Abogado de la Universidad Católica de Colombia, Magíster en Derecho (Summa Cum Laude) de la Universidad de Palermo y Doctor en Derecho (tesis distinguida) de la Universidad de Buenos Aires, área Filosofía del Derecho. Profesor de la Facultad de Derecho y Ciencias Políticas, Director del departamento de teoría jurídica y constitucional y Director de la Maestría en Derecho Constitucional de la Universidad de La Sabana, Colombia. Contacto: fabio.pulido@ unisabana.edu.co 


\section{RESUMEN}

En este trabajo se estudia la validez de los actos legislativos fast track(FT). Para ello, primero se analizará el concepto de validez jurídica y se resaltarán las nociones de pertenencia, aplicabilidad jurídica y aplicabilidad moral de las normas. Luego se mostrará que los FT no cumplen con dos condiciones necesarias para la validez de las reformas constitucionales en Colombia: su carácter democrático y la exigencia de rigidez constitucional. Se concluye que los FT solo pueden pretender aplicabilidad moral (por ejemplo, si cada una de sus normas son moralmente adecuadas) pero que, en ese sentido, esos mecanismos de reforma constitucional resultan jurídicamente irrelevantes.

Palabras clave: derecho constitucional; teoría del derecho; validezjurídica; proceso de paz; reforma constitucional 
ABSTRACT

This paper studies the legal validity of the fast track constitutional amendments (FT). First, the work analyses the concept of legal validity and highlights the next notions: membership, legal applicability and moral applicability of the legal rules. Later, the author argues that FT does not fulfill with two necessary validity conditions to constitutional amendments in Colombia: its democratic character and the constitutional rigidity. Lastly, it is concluded that FT only could claim moral applicability but in this sense FT are legally irrelevant.

Keywords: Constitutional law; jurisprudence; legal validity; peace process; constitutional amendment

SUMARIO

INTRODUCCIÓN. I. LA VALIDEZ DE LAS NORMAS JURÍDICAS. II. LA VALIDEZ DE LOS MECANISMOS DE REFORMA CONSTITUCIONAL EN LA CONSTITUCIÓN COLOMBIANA. A. Los procedimientos para la aprobación de los actos legislativos y los fast track. B. La democracia como condición de validez de los mecanismos de reforma. $C$. La rigidez constitucional como límite lógico o conceptual de las reformas constitucionales. CONCLUSIONES. BIBLIOGRAFÍA 


\section{INTRODUCCIÓN}

El 24 de noviembre de 2016, el Gobierno de la República de Colombia y las Fuerzas Armadas Revolucionarias de Colombia (FARC) suscribieron el Acuerdo Final para la terminación del conflicto y la construcción de una paz estable y duradera (en adelante "el Acuerdo"). El Congreso de la República, por su parte, aprobó el Acto Legislativo 1 de 2016 (en adelante AL) por medio del cual estableció los instrumentos jurídicos para la implementación del Acuerdo. ${ }^{1}$ En el artículo $1^{\circ}$ del AL se estableció un "procedimiento legislativo especial para la paz" con la intención de "agilizar y garantizar la implementación" del Acuerdo. Por medio de este mecanismo se definió un proceso abreviado para la aprobación de leyes y actos legislativos. En este contexto, se estableció un mecanismo abreviado de reforma constitucional: los actos legislativos fast track (en adelante FT). ${ }^{2}$

El problema que se aborda en este trabajo es el estatus jurídico de los FT, esto es, definir si la posibilidad de reformar la Constitución tal y como fue autorizada en el AL es válida. Para ello, primero se estudiará el concepto de validez jurídica y se resaltarán las nociones de pertenencia, aplicabilidad jurídica y aplicabilidad moral de las normas. Luego se mostrará que los FT no cumplen con dos condiciones necesarias para la validez de las

1 Colombia, Acto Legislativo 1 de 2016, por medio del cual se establecen instrumentos jurídicos para facilitar y asegurar la implementación y el desarrollo normativo del acuerdo final para la terminación del conflicto y la construcción de una paz estable y duradera, 49927 Diario Oficial, 7 de julio de 2016. http://www.secretariasenado.gov.co/senado/basedoc/acto_legislativo_01_2016.html

2 Ibid. Debe advertirse que la denominación fast track proviene del derecho constitucional estadounidense. De acuerdo con la constitución de los Estados Unidos, solo el Congreso puede aprobar las normas que rigen el comercio internacional. Al presidente, por su parte, le corresponde la negociación de esos acuerdos. Así, en los casos en que el presidente se comprometa en tratados que impliquen el cambio del orden jurídico interno, la implementación debe ser aprobada por el Congreso. Sin embargo, en estos casos el Congreso puede hacerlo mediante un procedimiento expedito conocido como fast track procedure (actualmente denominado Trade Promotion Authority), en el que, por ejemplo, el Congreso puede aprobar en bloque las medidas y sin posibilidad de incluirles modificaciones o enmiendas. Sobre el particular véase Harold Hongju Koh, The Fast Track and United States Trade Policy, 18 Brooklyn Journal of International Law, 1, 143-180 (1992) y José Julián Tole, Los TLC de Estados Unidos con países latinoamericanos: un modelo de integración económica "superficial" para el continente americano. Revista Derecho del Estado (30), 251-300. http://revistas.uexternado.edu.co/index. $\mathrm{php} / \mathrm{derest} /$ article/view/3525 
reformas constitucionales en Colombia: su carácter democrático y la exigencia de rigidez constitucional. Se concluye que los FT solo pueden pretender aplicabilidad moral (si cada una de las normas definidas en ellos resulta moralmente adecuada) pero que, en ese sentido, esos mecanismos de reforma constitucional resultan normativamente irrelevantes.

\section{LA VALIDEZ DE LAS NORMAS JURÍDICAS}

En el lenguaje jurídico el concepto de validez es empleado en dos sentidos principales: 1) validez como pertenencia de las normas jurídicas y 2) validez como carácter vinculante u obligatorio de las normas. ${ }^{3}$ De acuerdo con el primer sentido, una norma es válida cuando cumple con las condiciones de pertenencia establecidas en el ordenamiento jurídico. ${ }^{4}$ En un segundo sentido, se emplea el concepto de validez para referirse al carácter obligatorio o vinculante de las normas jurídicas. Según esto, afirmar que una norma es válida implica sostener que la conducta $\varnothing$, ordenada por la norma jurídica, es obligatoria. ${ }^{5}$ En estos términos, asegurar que una norma jurídica es válida (en el primer sentido) es lo mismo que afirmar que la norma pertenece a un ordenamiento jurídico. Y aseverar que una norma es válida (en el segundo sentido) significa que satisface las exigencias para la vinculatoriedad.

Ahora bien, en relación con la validez en el segundo sentido (validez como carácter vinculante), existen dos variables: 1) el "deber" (entrecomillado) de aplicar las normas puede referirse a la descripción de la existencia de ese deber. Es decir que, cuando

3 Eugenio Bulygin, Tiempo y Validez, en Análisis lógico y Derecho, 193-214 (C. Alchourron y E. Bulygin (Eds.) Centro de Estudios Constitucionales, Madrid, 1991). Un análisis más amplio de esta cuestión puede verse en W. Waluchow, Four Concepts of Validity: Reflections on Inclusive and Exclusive Positivism, en The Rule of Recognition and the United States Constitution, 123144 (en M. Adler and K. Himma eds., Oxford University Press, Oxford, 2009).

4 Eugenio Bulygin, nota al pie 26, 195-198. H. L. A. Hart, The concept of law (2nd ed. Ed. Penelope A. Bulloch and Joseph Raz, Clarendon Press, Oxford, 1994). Hans Kelsen, Teoría general del derecho y del Estado, trad. E. García Máynez, E., Trad. (5ª reimpresión de la 2a . ed., Universidad Nacional Autónoma de México, México, 1995). Hans Kelsen, Teoría pura del derecho, trad. R. Vernengo (14 ed., Editorial Porrúa, México, 2005). Ibid. 
se afirma -en este sentido-que se "debe" aplicar la norma N1 se está diciendo que existe una norma N2 (perteneciente al ordenamiento jurídico) que establece el deber de aplicar N1. y 2) el deber (desentrecomillado) de aplicarlas se refiere a la afirmación de que existe un deber de obedecer, seguir o aplicar la norma N1. En este caso, el deber de aplicar la norma N1 depende de la existencia de un fundamento moral para obedecer N1. Para mayor claridad en la exposición, se denominará a la primera variable aplicabilidad jurídica o positiva de las normas y a la segunda aplicabilidad moral de las normas. Ahora bien, en la explicación de la aplicabilidad jurídica de las normas, los teóricos del derecho han diferenciado entre pertenencia y aplicabilidad. ${ }^{6}$ Una norma $\mathrm{N}$, como se dijo, pertenece a un ordenamiento jurídico OJ si y solo si cumple con las condiciones de validez definidas en OJ. Por otro lado, $\mathrm{N}$ es aplicable si y solo si existe una norma valida (perteneciente) N2 en OJ que establece el "deber" (entrecomillado) de seguir o aplicar N. ${ }^{7}$ Esta distinción muestra algo importante: no toda norma perteneciente es aplicable, ni toda norma aplicable es perteneciente. Por ejemplo, una norma que no ha sido aprobada de conformidad con las condiciones institucionales de validez (por ejemplo, no se surtieron los debates necesarios) no pertenece al ordenamiento jurídico. Sin embargo, puede ocurrir que, en virtud de otra norma perteneciente, se establezca el deber de aplicar tales normas (como la regla que establece la presunción de constitucionalidad de las leyes).

Otro complejo problema que surge de este análisis es definir el tipo de relaciones que existen entre la validez -en el primer sentido- y la aplicabilidad moral de las normas. El hecho de que una norma pertenezca a un ordenamiento jurídico (es decir, que cumpla con las condiciones de validez) no significa que

6 Pablo Navarro y Jorge Rodríguez, Deontic Logic and Legal Systems 132-136 (Cambridge University Press, New York, 2014.

7 La cuestión de en qué sentido se puede afirmar que validez implica obligatoriedad ha sido estudiada por Joseph Raz, The Authority of Law Essays on Law And Morality 148-159 (Oxford University Press, Oxford, 1979) y Carlos Santiago Nino, La validez del derecho 7-28 (Editorial Astrea, Buenos Aires, 1986). Ambos autores parten desde un punto de vista crítico de la tesis de Kelsen quien considera que una norma válida (perteneciente) es necesariamente vinculante (Hans Kelsen, supra nota 4, 23-28). 
necesariamente tenga fuerza vinculante. Esto es así toda vez que "[d]el hecho de que algo es no puede inferirse que algo deba ser". ${ }^{8}$ Por ello, para que las normas tengan fuerza vinculante es necesario que tengan soporte moral, que puede provenir de tres vías: 1) cuando se muestre que dentro de las condiciones de validez (como pertenencia) de las normas se contemplen criterios morales (por ejemplo que dentro de esas condiciones se defina que estas deben ser consistentes con los derechos humanos de las personas); 2) cuando el fundamento de los criterios de validez (aun cuando no ellos mismos) sea de naturaleza moral (o sea, que se admita que para que una norma sea válida deba provenir necesariamente de una autoridad moral) y 3) cuando la norma -por sí misma- tiene contenido moral (es decir, coincide con lo que exige la moral). No es posible, por razones de espacio, desarrollar en este trabajo todo el alcance de este complejo tema. Basta mencionar que solo un concepto normativo de validez (que logra explicar el vínculo entre pertenencia y aplicabilidad moral) es relevante para dar cuenta del valor práctico del derecho (como la capacidad del derecho de constituirse en razones justificatorias para la acción), pues, de lo contrario, no se lograría explicar en qué casos el derecho es genuinamente vinculante (es decir, cuándo sus normas son capaces de constituirse en deberes). Pero al mismo tiempo esta explicación debe mostrar de qué manera el derecho hace diferencia práctica con la moral y no se torna superfluo respecto de ella. En otras palabras, debe mostrar cómo es capaz de ser fuente de razones jurídicas justificadoras para la acción, pero que ellas no colapsan en las razones morales ni simplemente las reiteran. $\mathrm{Si}$, por ejemplo, se afirma que el derecho es vinculante si y solo si su contenido coincide con la moral, entonces, las reglas jurídicas son superfluas (y, por tanto, no cumplen ningún rol) en el razonamiento justificatorio de los individuos y operadores jurídicos. ${ }^{9}$

8 Carlos Santiago Nino, La validez del derecho 11 (Editorial Astrea, Buenos Aires, 1986).

9 Acerca del problema de la superfluidad del derecho véase Joseph Raz, Reasoning with rules, 54, Current Legal Problems, 1-18 (2001); Carlos Santiago Nino, El constructivismo ético 113133 (Centro de Estudios Constitucionales, Madrid, 1989), y Juan Cianciardo, La paradoja de la irrelevancia moral del gobierno y del Derecho. Una aproximación desde el pensamiento de 
Tabla 1. Los sentidos de validez

\begin{tabular}{|l|l|}
\hline Pertenencia & $\begin{array}{l}\text { N1 es válida si y solo si existe una } \\
\text { N2 que autoriza al órgano O, } \\
\text { mediante el procedimiento P, para } \\
\text { que expida N1 con el contenido C. }\end{array}$ \\
\hline $\begin{array}{l}\text { Aplicabilidad } \\
\text { jurídica }\end{array}$ & $\begin{array}{l}\text { N1 es aplicable si y solo si existe una N2 } \\
\text { válida (perteneciente) que exige aplicar N1. }\end{array}$ \\
\hline $\begin{array}{l}\text { Aplicabilidad } \\
\text { moral }\end{array}$ & $\begin{array}{l}\text { N1 tiene fuerza vinculante si y solo si existe } \\
\text { una norma moral que exija aplicar N1. }\end{array}$ \\
\hline
\end{tabular}

Para terminar este apartado veamos brevemente la cuestión acerca de la declaración judicial de validez de normas inválidas. En general, la declaración judicial no es una condición de validez de las normas jurídicas (salvo, por ejemplo, cuando se establece control constitucional previo). Pero más importante aún es anotar que las condiciones de validez son objetivas pues son independientes de las consideraciones subjetivas de los operadores jurídicos en general y de los jueces en particular. En consecuencia, que un juez (así sea un órgano de cierre) concluya que una norma es válida (o aplicable jurídicamente) no significa que necesariamente así lo sea. Para abordar este problema Hart propuso diferenciar entre la definitibilidad y la infalibilidad de las decisiones judiciales. El hecho de que un órgano de cierre (o, en general, un juez cuya decisión tiene efectos de cosa juzgada) tenga la última palabra sobre la aplicación del derecho, hace que sus decisiones sean jurídicamente indiscutibles. Pero obviamente el carácter definitivo de las decisiones judiciales no las libera de incurrir en errores (como la identificación o aplicación de las condiciones de validez). Para explicar esta diferencia, Hart recurre al ejemplo de los juegos. Una de sus propiedades es su carácter reglado, es decir, la definición de movidas válidas e inválidas mediante reglas. Muchos juegos, además, incluyen

Carlos Nino, 19 Dikaion, $47-70$ (2010) b. http:/dikaion.unisabana.edu.co/index.php/dikaion/ article/view/1702 
árbitros que, entre otras cosas, tienen la competencia para decidir si se logró o no un punto en una jugada específica. Esto significa que los juegos que contemplan árbitros incluyan una regla que establece que sus decisiones (incluso las equivocadas) se deben considerar vinculantes para las partes. Pero el hecho de que exista esta regla, no significa que los árbitros no se puedan equivocar ni que, por supuesto, cuando lo hagan modifiquen las reglas del juego. En el caso del fútbol, el árbitro tiene la última palabra sobre lo que es un gol; sin embargo, eso no lo exime de cometer errores honestos o de violar maliciosamente su deber de aplicar la norma. Lo importante en estos asuntos es evidenciar que la regla que les da voz definitiva a ellos no significa que les otorgue la autoridad para modificar las reglas del juego ni para cambiar las condiciones de validez, esto es, los criterios a partir de los cuales se define si una regla es o no parte del juego. ${ }^{10}$

Como se puede ver, es posible que una norma inválida sea considerada aplicable en virtud de la regla que otorga el carácter definitivo a las decisiones judiciales. Los ordenamientos jurídicos, como se dijo, están conformados por un conjunto de normas que comparten en común los criterios de validez (de pertenencia). Pero además, esos ordenamientos son abiertos en el sentido de que autorizan la aplicación de normas no pertenecientes. Al igual que los árbitros de fútbol, los jueces cometen errores. Y al igual que las reglas del fútbol, los ordenamientos jurídicos exigen que las decisiones de los jueces sean vinculantes (aplicables jurídicamente) aun cuando se basen en argumentos inválidos. En resumen, una decisión judicial definitiva puede autorizar la aplicación de una norma inválida, pero no significa que esa norma sea válida ni que en el ordenamiento jurídico se hayan modificado las condiciones de validez.

10 H. L. A. Hart, supra nota 4, 176-177. Una revisión de la tesis de Hart puede verse en Andrej Kristan, A paradox of Hart's Fallible Finality, 2016, Analisi e diritto, 339-344 (2016). 


\section{LA VALIDEZ DE LOS MECANISMOS DE REFORMA CONSTITUCIONAL EN LA CONSTITUCIÓN COLOMBIANA}

En este apartado se presentarán las condiciones de validez de los mecanismos de reforma constitucional en la Constitución colombiana, mostrando, en primer lugar, las características procedimentales en la aprobación de esos actos legislativos y sus diferencias principales con el procedimiento de los FT. Luego se estudiarán dos condiciones de validez específicas de los mecanismos de reforma constitucional: el carácter democrático de los procedimientos y la rigidez constitucional.

\section{A. Los procedimientos para la aprobación de los actos legislativos y los fast track}

El artículo 374 establece que la Constitución Política puede ser reformada por el Congreso (mediante actos legislativos), "por una Asamblea Constituyente o por el pueblo mediante referendo"." Específicamente, para la creación de actos legislativos, el artículo 375 de la Constitución Política contempla dos fases en el procedimiento:

1. La iniciativa normativa, esto es, la facultad atribuida a diferentes actores para que concurran a la presentación de proyectos normativos (proyectos de ley o de reforma constitucional) ante las autoridades para que realicen los trámites constitucionales, legales y reglamentarios que correspondan. ${ }^{12}$ Para el caso de los actos legislativos, la Constitución colombiana precisa que tienen facultad de iniciativa normativa: 1) el Gobierno nacional; 2) diez miembros del Congreso; 3) el $30 \%$ de los concejales o de

11 Colombia, Constitución Política, versión corregida, 116 Gaceta Constitucional, 20 de julio de 1991, artículo 374. http://www.secretariasenado.gov.co/senado/basedoc/constitucion_politica_1991_pr012.html \#375

12 Alfonso Palacios Torres, Concepto y control del procedimiento legislativo (1 ed., Universidad Externado de Colombia, Bogotá, 2005). Juan C. Lancheros, María Mantilla, Fabio Pulido Ortiz, Ingrid Suárez y Rocío Rincón, Hoja de Ruta. Trámite legislativo ordinario (Konrad Adenauer Stiftung, Bogotá, 2010). 
los diputados ${ }^{13}$ y 4) el $5 \%$ del censo electoral vigente. ${ }^{14}$ Es necesario hacer dos precisiones en torno a la iniciativa normativa de actos legislativos. Primero, a diferencia de lo que ocurre en el procedimiento de creación de leyes, el Gobierno nacional no tiene iniciativa normativa reservada para el trámite de los actos legislativos..$^{15}$ Incluso, la Corte Constitucional ha sostenido que el fundamento de la iniciativa privativa del Gobierno es evitar que se legisle sin su conocimiento y consentimiento en materias que comprometen aspectos propios de su competencia. ${ }^{16}$ Segundo, la iniciativa popular normativa "es un derecho político de un grupo de ciudadanos de presentar proyectos de acto legislativo y de ley ante el Congreso, de ordenanza ante las asambleas departamentales, de acuerdos ante los concejos municipales y de resolución ante las juntas administradoras locales". ${ }^{17} \mathrm{La}$ Corte Constitucional ha enfatizado que la iniciativa popular, mucho más si se trata de proyectos de reforma constitucional "tiene la naturaleza de un derecho político fundamental de origen cons-

13 Debe tenerse en cuenta que sobre este asunto existen dos normas constitucionales contradictorias. El artículo 155 establece el $30 \%$ de concejales o diputados (Colombia, Constitución Política, supra nota 5). Por otra parte, el artículo 375 define que el $20 \%$ de concejales o diputados (Colombia, Constitución Política, supra nota 5, artículo 375). Para la Corte Constitucional "pese a la aparente contradicción entre los dos preceptos, se refieren en realidad a modalidades distintas de procesos modificatorios de la Carta: mientras el primero de ellos, que exige el 30 $\%$ de los concejales o diputados, toca genéricamente con proyectos de reforma constitucional, el 375 alude de manera específica a proyectos de acto legislativo, es decir, a los que tramita el Congreso, de lo cual se desprende que la segunda disposición es especial para ese procedimiento de enmienda, por lo que la otra queda reservada para los casos contemplados en los artículos 376 y 378" (Corte Constitucional, Sala Plena, Sentencia C-222-97, Expediente D-1465, Magistrado Ponente: José Gregorio Hernández).

14 Colombia, Constitución Política, supra nota 11, artículo 375.

15 En materia de leyes, el Gobierno puede presentar proyectos de ley sobre todas las materias y, adicionalmente, tiene potestad exclusiva para presentar proyectos en las siguientes materias: a) Plan Nacional de Desarrollo; b) Ley anual de presupuesto; c) Banco de la República y funciones de competencia para su junta directiva; d) regulación del comercio exterior y fijación del régimen de cambio internacional; e) exenciones de impuestos, contribuciones o tasas nacionales; $f$ ) organización, administración, control y explotación de los monopolios rentísticos que estarán sometidos a un régimen propio; g) aprobación de los tratados o convenios que el Gobierno celebre con otros Estados o con entidades de Derecho Internacional y h) facultades extraordinarias. Constitución Política, supra nota 11, Artículos 375 y 154. Colombia, Artículo 142 de la Ley $5^{a}$ de 1992, por la cual se expide el Reglamento del Congreso, el Senado y la Cámara de Representantes, Diario Oficial 40.483, 18 de junio de 1992. http://www.secretariasenado.gov. co/index.php/ley-5-de-1992

16 Corte Constitucional, Sala Plena, Sentencia C-1707-00, Expediente OP-037. Magistrado Ponente: Cristina Pardo Schlesinger.

17 Corte Constitucional, Sentencia C-150-15, Sala Plena, Expediente PE-038. Magistrado Ponente: Mauricio González Cuervo. 
titucional, atribuido a todo ciudadano, con miras a que pueda participar en la conformación, ejercicio y control político". ${ }^{18}$

2. Luego de su presentación, el proyecto de Acto Legislativo debe publicarse en la Gaceta del Congreso para entonces iniciar el trámite parlamentario en la Comisión respectiva. ${ }^{19}$ El trámite parlamentario de los proyectos de acto legislativo tiene las siguientes características: 1) debe tramitarse "en dos periodos ordinarios y consecutivos"; ${ }^{20}$ 2) aprobado en el primer periodo, mediante mayoría simple, el proyecto debe ser publicado por el Gobierno y su aprobación en el segundo periodo exige mayoría absoluta";21 3) en el segundo periodo "solo podrán debatirse iniciativas presentadas en el primero; ${ }^{22} 4$ ) el Congreso de la República, en desarrollo del principio democrático y de la representación, puede incluir reformas a los proyectos de acto legislativo siempre que se garanticen los principios de unidad de materia, de consecutividad y de identidad; ${ }^{23}$ y 5) para asegurar una adecuada deliberación y protección de las minorías parlamentarias, no es posible la votación en bloque ni la realización de sesiones conjuntas. ${ }^{24}$

Estas exigencias procedimentales, como lo ha reconocido la Corte Constitucional, encuentran sustento en el modelo de democracia reconocido en la Constitución colombiana. Según la Corte:

18 Corte Constitucional sentencias C-180-94, Sala Plena, Expediente PE-005. Magistrado Ponente: Hernando Herrera Vergara y Corte Constitucional, supra nota 17.

19 Colombia, Constitución Política, supra nota 11 y Colombia, Ley $5^{\text {a }}$ de 1992, supra nota 15.

20 Esto es en los dos periodos definidos en el artículo 139 de la Constitución: "El primer periodo de sesiones comenzará el 20 de julio y terminará el 16 de diciembre; el segundo el 16 de marzo y concluirá el 20 de junio". Colombia, Constitución Política, supra nota 15, artículo 138.

21 Ibid., artículo 375.

22 Ibid.

23 Ibid. De acuerdo con el principio de unidad de materia se debe garantizar que el proyecto tenga un tema central que se vincula con cada una de sus normas. El de consecutividad exige que el trámite debe hacerse de manera sucesiva y en el orden constitucional establecido y el de identidad que las modificaciones deben tener un vínculo o conexidad con los temas previamente debatidos. Sobre la importancia de estos principios en el procedimiento de reformas constitucionales, véase Corte Constitucional, Sentencia C-140-10, Sala Plena, Expediente D-3075. Magistrado Ponente: Humberto Sierra Porto.

24 Ibid. y Corte Constitucional Sentencia C-387-97, Sala Plena, Expediente D-1545. Magistrado Ponente: Fabio Morón Díaz. 
[1]as reglas son inherentes a la democracia: reglas sobre las mayorías y minorías, reglas sobre las elecciones y la actuación de los parlamentos, reglas sobre las condiciones de ejercicio de la libertad política y, sobre todo, reglas sobre la protección de ciertos intereses básicos de todos los seres humanos..$^{25}$

La existencia de reglas procedimentales "es una característica distintiva del modelo democrático, y se manifiesta desde la elección de los representantes, hasta el producto final de la actuación de estos". ${ }^{26}$ De esta manera, esas reglas no son -y no deben serun simple capricho formalista, sino que deben estar diseñadas para mejorar la posibilidad de que el marco institucional sirva de presupuesto para alcanzar respuestas imparciales. ${ }^{27}$ Por tanto:

[1]as reglas procedimentales previstas para la implementación de la toma de decisiones son fundamentales a la hora de reconocer la validez tanto al procedimiento inicial, cuya finalidad es concitar el apoyo ciudadano a una propuesta de reforma, como la actuación final que pone término al proceso de reforma. ${ }^{28}$

Además, la particular exigencia en el cumplimiento de las reglas procedimentales para la aprobación de actos legislativos es una manifestación del principio de rigidez constitucional. Más adelante se explicará que la rigidez constitucional está en función de dos variables: el órgano de producción y el procedimiento de reforma constitucional. De acuerdo con la primera variable, la rigidez está en función de la posibilidad de diferenciar entre los órganos con poderes legislativos ordinarios y los órganos con poder de reforma constitucional. De acuerdo con la segunda, la rigidez constitucional supone que las reformas constitucionales deben hacerse mediante procedimientos más exigentes y complejos que los necesarios para crear normas infraconstitucionales.

\footnotetext{
25 Corte Constitucional, supra nota 23.

26 Ibid.

27 La importancia de la institucionalización de los procedimientos ha sido resaltada por Carlos Santiago Nino, La constitución de la democracia deliberativa, traducción Roberto P. Saba (Gedisa, Barcelona, 2003). Sobre este asunto véase también Facundo García Valverde, Agencia política y legitimidad en la democracia deliberativa, 22, Eidos (2015), 225-252. http://www. scielo.org.co/scielo.php?script=sci_arttext\&pid=S1692-88572015000100011

28 Corte Constitucional, supra nota $2 \overline{3}$.
} 
En conclusión, el cumplimiento de las reglas procedimentales para la aprobación de los actos legislativos es una condición necesaria para la validez de las reformas a la Constitución por esta vía. Primero, porque esas reglas son necesarias para asegurar el carácter democrático de las reformas. Segundo, porque las exigencias procedimentales constituyen una condición necesaria para la rigidez constitucional.

Veamos ahora brevemente las particularidades del procedimiento de reforma constitucional abreviado establecido en el artículo $1^{\circ}$ del Acto Legislativo (AL) y sus principales diferencias con el procedimiento de aprobación de los actos legislativos (véase tabla 2). De acuerdo con esa disposición constitucional la aprobación de los FT contempla las siguientes reglas: 1) los proyectos deben tener iniciativa exclusiva del Gobierno nacional y "solo podrán tener modificaciones siempre que se ajusten al contenido del Acuerdo Final y que cuenten con el aval previo del Gobierno nacional". Esto significa que, a diferencia de los actos legislativos, para el caso de los FT se establece una reserva de iniciativa en cabeza del Gobierno nacional y, por tanto, se suspende el derecho político fundamental reconocido en el artículo 40 de la Constitución. Además, el hecho de que las iniciativas normativas solo puedan provenir del Gobierno nacional, le otorga a este órgano del Estado competencias de reforma constitucional; 2) el contenido del proyecto de acto legislativo FT debe tener "por objeto facilitar y asegurar la implementación y desarrollo normativo del Acuerdo Final para la terminación del conflicto y la construcción de una paz estable y duradera";29 3) los proyectos de FT tienen trámite preferencial y preferencia absoluta "en el Orden del Día sobre cualquier otro asunto, hasta tanto la respectiva Cámara o Comisión decida sobre ellos;" 4) el título de los FT debe "corresponder precisamente a su contenido" y debe contemplar la siguiente fórmula: "El Congreso de Colombia, en virtud del Procedimiento Legislativo Especial para la Paz, DECRETA"; 5) el primer debate "se surtirá en sesión conjunta

29 Colombia, Acto Legislativo, supra nota 1. 
de las Comisiones Constitucionales Permanentes respectivas"; 30 6) los FT deben ser tramitados en cuatro debates, en una sola vuelta (es decir, uno de los periodos contemplados en el artículo 139 de la Constitución), deben ser aprobados con mayoría absoluta y podrán tramitarse en sesiones extraordinarias (recuérdese que el trámite de los actos legislativos exige ocho debates (sin sesiones conjuntas) y dos periodos ordinarios y consecutivos); 7) el trámite de los FT autoriza que "[e]n la comisión y en las plenarias se decidirá sobre la totalidad de cada proyecto, con las modificaciones avaladas por el Gobierno nacional, en una sola votación";31 8) una vez aprobados, la Corte Constitucional debe revisar de forma automática los FT. ${ }^{32}$

Tabla 2. Principales diferencias entre los procedimientos de aprobación de los actos legislativos y los FT.

\begin{tabular}{|l|l|l|}
\hline & Actos Legislativos & Fast Track \\
\hline Iniciativa & - Gobierno nacional & - Exclusiva del \\
normativa & (sin cláusula & Gobierno \\
& de reserva) & \\
& - Congreso & \\
& - Ciudadana & \\
\hline
\end{tabular}

30 Debe tenerse en cuenta que, con el propósito de asegurar la debida deliberación, la Corte Constitucional ha sostenido que "la aprobación de los actos legislativos requiere los ocho debates previstos por la Carta, por lo cual no es posible la realización de sesiones conjuntas de las comisiones permanentes para la aprobación de actos legislativos". Corte Constitucional Sentencia C-387-97, Sala Plena, Expediente D-1545. Magistrado Ponente: Fabio Morón Díaz.

31 La Corte Constitucional ha excluido del trámite de los actos legislativos las votaciones en bloque por considerarlas contrarias a la protección de las minorías y a la debida deliberación necesaria para la aprobación de reformas constitucionales. Corte Constitucional Sentencias supra nota 17 y supra nota 16 .

32 Colombia, Acto Legislativo supra nota 1, Artículo 1 literal K. Obsérvese que a diferencia de las leyes estatutarias, el control constitucional de los FT no es un elemento del procedimiento pues, como lo establece el AL, el control constitucional es control "posterior a su entrada en vigencia". 


\begin{tabular}{|c|c|c|}
\hline & Actos Legislativos & Fast Track \\
\hline Debates & $\begin{array}{l}\text { - Ocho debates } \\
\text { - Sin comisiones } \\
\text { conjuntas ni sesiones } \\
\text { extraordinarias. } \\
\text { - Dos periodos } \\
\text { ordinarios y } \\
\text { consecutivos. } \\
\text { - Sin trámite } \\
\text { de urgencia. }\end{array}$ & $\begin{array}{l}\text {-Cuatro debates } \\
\text { - Comisiones } \\
\text { conjuntas y sesiones } \\
\text { extraordinarias. } \\
\text { - Con trámite } \\
\text { de urgencia. } \\
\text { - Un periodo } \\
\text { ordinario. }\end{array}$ \\
\hline Modificaciones & $\begin{array}{l}\text { - Facultad del } \\
\text { Congreso }\end{array}$ & $\begin{array}{l}\text { - Aval previo del } \\
\text { Gobierno nacional. }\end{array}$ \\
\hline Votaciones & $\begin{array}{l}\text { - Prohibición de } \\
\text { votaciones en bloque. }\end{array}$ & Votaciones en bloque \\
\hline
\end{tabular}

\section{B. La democracia como condición de validez de los mecanismos de reforma}

La Constitución colombiana se caracteriza por reconocer el principio democrático como fundamento de su organización política y como parte esencial de los derechos políticos fundamentales de los ciudadanos. ${ }^{33}$ A hora bien, el reconocimiento de ese principio no se reduce a la validación de los triunfos mayoritarios en elecciones y en cuerpos colegiados. La democracia es en realidad un método para alcanzar respuestas políticas correctas e imparciales. Lo que se pretende con los modelos democráticos es institucionalizar los ideales de la deliberación racional: el intercambio genuino de ideas, el acceso a diferentes puntos de vista, el conocimiento y rectificación de la infor-

33 Desde el preámbulo, la Constitución colombiana elige a la democracia como fundamento del ordenamiento jurídico. El artículo $1^{\circ}$, por su parte, afirma que el Estado colombiano se organiza con base en una República democrática. El artículo 40 reconoce una serie de derechos que tiene como fundamento el carácter democrático de la Constitución, el artículo 103 desarrolla los mecanismos de participación democrática y el artículo 95 contempla como deber de los ciudadanos "[r]espetar y apoyar a las autoridades democráticas legítimamente constituidas". 
mación (fáctica y normativa) relevante, la depuración de los errores y falencias fácticos, lógicos y sustantivos, la ampliación de diferentes argumentos y la identificación de soluciones alternativas. ${ }^{34}$ En otros términos, la justificación de la democracia reside en su capacidad epistémica sobre las exigencias del bien común, puesto que la deliberación intersubjetiva hace que los individuos intercambien creencias, ideas y justificaciones para constituirse de esa manera en un procedimiento confiable para lograr soluciones justas puesto que:

[e]l intercambio de ideas y la necesidad de ofrecer justificaciones frente a los otros no solo incrementa el conocimiento que uno posee y detectar defectos en el razonamiento, sino que ayuda a satisfacer el requerimiento de atención imparcial a los intereses de todos los afectados. ${ }^{35}$

En este orden de ideas, existen diferentes elementos que la deliberación reproduce y que le confieren el carácter epistémico a la democracia. Entre ellos resaltan los siguientes: 1) la participación en la discusión de todas las partes interesadas y potencialmente afectadas; 2) participación en el debate en condiciones de igualdad y libertad; 3) expresión y justificación genuina de razones e intereses y 4) depuración mediante la discusión y el diálogo de falencias fácticas, lógicas y sustantivas. De acuerdo con esto, el principio democrático exige que los diseños institucionales de toma de decisiones políticas implementen los presupuestos de la deliberación. ${ }^{36}$ Se supone que los sistemas democráticos ponen en marcha un conjunto de reglas que formalizan las condiciones ideales de razonamiento y deliberación públicos, de manera tal que en la configuración del diseño institucional de la democra-

34 David Estlund, La autoridad democrática. Los fundamentos de las decisiones políticas legítimas, trad. Sara Palacio Gaviria y Sebastián Linares (Siglo XXI Editores, Buenos Aires, 2011). En La constitución de la democracia deliberativa, traducción Roberto P. Saba (Gedisa, Barcelona, 2003), Carlos Santiago Nino resalta la importancia de la institucionalización de los procedimientos. Sobre este asunto véase también Facundo García Valverde, Agencia política y legitimidad en la democracia deliberativa, 22, Eidos (2015), 225-252. http://www.scielo.org. co/scielo.php?script=sci_arttext\&pid=S1692-88572015000100011

35 Carlos Nino, supra nota $34,160-161$.

36 Ibid. Horacio Spector, Democracia y control constitucional: una tensión aparente, en homenaje a Carlos Nino, 231-247 (M. Alegre, et. al., La Ley, Buenos Aires, 2008). 
cia son fundamentales las reglas de procedimiento de toma de decisiones públicas. ${ }^{37}$

La Corte Constitucional ha reconocido la importancia de estas reglas -en especial para los mecanismos de reforma-. En la Sentencia C-141 de 2010, el Alto Tribunal declaró la inconstitucionalidad de una ley que convocaba a referendo para la reforma de la Constitución por presentarse diferentes irregularidades en su trámite. Igualmente, en esa sentencia estudió la constitucionalidad del trámite ante la organización electoral de la iniciativa legislativa ciudadana que dio origen a la Ley 1354 de 2009 (convocatoria de referendo). La Corte Constitucional debía resolver si la violación de los topes individuales y globales establecidos para la campaña de iniciativa legislativa popular llevaba a la inconstitucionalidad de la ley. Algunos defensores de la convocatoria a referendo argumentaron que 1) con independencia de los vicios de procedimiento, la recolección de firmas en la iniciativa ciudadana era prueba suficiente de la voluntad popular y 2) los requisitos y formalidades de esas campañas no estaban explícitamente consagradas en la Constitución. No obstante, la Corte Constitucional desestimó estos argumentos fundamentándose principalmente en el especial valor que tiene la regulación del procedimiento democrático en la formación de los actos de reforma constitucional. Así, para la Corte, "la elección constitucional de la forma de gobierno democrática determina el diseño institucional del Estado colombiano" y en ese orden de ideas, el carácter reglado es una característica esencial de la toma de decisiones en una democracia:

[y] se manifiesta desde la elección de los representantes, hasta el producto final de la actuación de estos. Las reglas procedimentales constituyen por tanto un instrumento para la consecución de los valores sustanciales perseguidos mediante la actuación democrática, pero a su vez, por su importancia, adquieren un valor sustantivo. ${ }^{38}$

37 Jürgen Habermas, Facticidad y validez 172 (Trotta, Madrid, 1998). En palabras de Nino, el procedimiento democrático es el producto de reglas que "no son arbitrarias sino que están diseñadas para maximizar el valor epistémico de aquel proceso". Carlos Nino, supra nota 34, 271.

38 Corte Constitucional sentencias, supra nota 23. La importancia de la democracia deliberativa 
En consecuencia, el reconocimiento del principio democrático en la Constitución colombiana impacta, como lo sostiene la Corte Constitucional, en la validez de la implementación de los diseños de toma de decisiones públicas y en particular en los procedimientos definidos para los mecanismos de reforma constitucional. Esos procedimientos, en tanto tendrán como resultado las decisiones a las cuestiones fundamentales de la organización pública, deben ser más sensibles en la implementación de los presupuestos de la democracia. ${ }^{39}$

No obstante, existen diferentes aspectos que afectan la justificación democrática de las reformas constitucionales por medio de los FT y que, por tanto, siembran dudas acerca de su validez constitucional. En primer lugar, el hecho de que todas las propuestas normativas estén monopolizadas por el Gobierno nacional restringe indebidamente las posibles alternativas a los problemas que se pretendan abordar mediante los $\mathrm{FT}^{40}$ Recuérdese que tanto las iniciativas normativas, como las enmiendas en el trámite, son exclusivas del Gobierno nacional. Con ello se bloquean las eventuales propuestas que puedan surgir en la deliberación de los proyectos y se restringe el derecho constitucional fundamental de iniciativa legislativa. En segundo lugar, la calidad del debate es evidentemente débil para justificar el carácter democrático de las reformas constitucionales. En

en el derecho constitucional colombiano la muestra Leonardo García Jaramillo, Recepción de postulados deliberativistas en la jurisprudencia constitucional. Análisis crítico a partir del tribunal colombiano, 10, Revista Argentina de Teoría Jurídica (2008). http://www.utdt.edu// ver_contenido.php?id_contenido $=2450 \& i d$ item_menu $=3555$

39 La importancia en el diseño de procedimientos democráticos en los mecanismos de reforma constitucional ha sido estudiado y resaltado por Stephen Tierney, Constitutional Referendums. The Theory and Practice of Republican Deliberation (Oxford: Oxford University Press, 2012). Un estudio de esta cuestión en el proceso de paz en Colombia puede verse en R. Gargarella, Pensar sobre la democracia, discutir sobre los derechos. 267, Nueva Sociedad (2017) 101-113; y en F. Pulido Ortiz y G. Bustos, Momentos constitucionales y legitimidad de los procedimientos de reforma, en procesos legislativos y ordenamiento constitucional: abordajes teóricos, 92-105 (Ed. G. Eljach, CAEL, Bogotá, 2016).

40 No sobra advertir que, además, este monopolio se torna aún más problemático al constituirse en una reforma de carácter hiperpresidencialista que pretende "combatir o morigerar el hiperpresidencialismo, que se identificaba como causa fundamental de la inestabilidad política" en Latinoamérica. Sobre el tema véase Roberto Gargarella y Christian Courtis, El nuevo constitucionalismo latinoamericano: promesas e interrogantes 10 (Santiago de Chile, Cepal, 2009). http://repositorio.cepal.org/bitstream/handle/11362/6162/1/S0900774_es.pdf 
realidad el debate exigido en los FT, no solo es inferior al de los mecanismos ordinarios de reforma, sino que es inferior o igual al de la legislación: se requieren solamente cuatro debates (lo mismo que se exige, por ejemplo, para una ley ordinaria), y se autorizan las sesiones conjuntas, el trámite de urgencia y las votaciones en bloque. De esta forma, las exigencias deliberativas para los FT son, como mucho, iguales de exigentes a las que se requieren para aprobar, por ejemplo, leyes estatutarias o leyes orgánicas. Incluso, es posible afirmar que las exigencias deliberativas son más fuertes para el caso de las leyes estatutarias toda vez que el trámite de este tipo de leyes requiere que se desarrolle una legislatura y contempla como parte del trámite el control previo de la constitucionalidad del proyecto (recuérdese que el de los FT es control posterior).

En suma, la restricción en las iniciativas normativas, la reducción en número y calidad de los debates y la obligación de aprobar los proyectos mediante votaciones en bloque y en corto tiempo, reduce sustancialmente la capacidad del procedimiento para lograr resultados legítimos e imparciales de la forma como lo exige el principio democrático. De esta manera, los actos de reforma FT, en tanto no cumplen con las exigencias deliberativas mencionadas, no son mecanismos de reforma constitucional válidos (es decir, no cumplen con las condiciones de pertenencia al ordenamiento jurídico colombiano). Debe observarse que el principio democrático es un principio moral que otorga legitimidad a determinados procedimientos legislativos y constitucionales (es una condición de aplicabilidad moral de las reformas constituciones). ${ }^{41}$ Pero, además, en el caso colombiano el principio democrático es una condición de pertenencia de las normas que pretenden reformar la Constitución. ${ }^{42}$ Es cierto que la implementación de este principio en instituciones específicas no es una tarea sencilla, pero también lo es que la reducción de las exigencias deliberativas en los mecanismos de reforma cons-

41 Carlos Nino, supra nota 34.

42 Corte Constitucional sentencias, supra nota 23. Como se verá más adelante tampoco existen razones para pensar que los FT, a pesar de ser inválidos, resulten aplicables. 
titucional, a tal punto que los lleve a estar al mismo nivel de la legislación, desconoce el requisito de rigidez que demandan los mecanismos de reforma en la Constitución colombiana.

\section{La rigidez constitucional como límite lógico o conceptual de las reformas constitucionales}

El poder constituyente, en términos amplios, es aquel que tiene la autoridad para crear normas constitucionales. Tradicionalmente se ha distinguido entre el poder constituyente originario (por ejemplo, el poder de creación de una nueva Constitución) y el poder constituyente derivado (como el poder de reformar la Constitución). ${ }^{43}$ Uno de los problemas centrales que deben abordarse al caracterizar e implementar el poder constituyente derivado es los límites en la competencia de reforma. ${ }^{44}$ Existen, en primer lugar, límites constituidos por los requerimientos morales acerca de las normas que deben o no crear los poderes de reforma. ${ }^{45}$ Obsérvese que este tipo de límites está asociado a la noción de aplicabilidad moral de las normas jurídicas explicadas más arriba. Según esto, una reforma constitucional tendrá fuerza (moral) vinculante si y solo si existen normas morales que exijan la creación de esa reforma. Y, por lo mismo, en relación con una reforma que esté en contra de esas normas morales no se puede predicar fuerza vinculante. ${ }^{46}$ En segundo

43 M. del Rosario-Rodríguez, La supremacía constitucional: naturaleza y alcances, 20 Díkaion 97-117 (2011). http://dikaion.unisabana.edu.co/index.php/dikaion/article/view/1950/2506

44 Rodrigo González Quintero, Procesos Constituyentes y Cortes Constitucionales: una perspectiva comparada, 18 Díkaion, 135-161 (2009). http://dikaion.unisabana.edu.co/index.php/ dikaion/article/view/1546/1868

45 Debe anotarse que la distinción entre límites jurídicos y morales, no es óbice para admitir que los ordenamientos jurídicos puedan reconocer principios morales como parte de los criterios de validez jurídica de las reformas (por ejemplo, el principio democrático en la Constitución colombiana explicado en el apartado anterior). Asimismo, debe señalarse que en la teoría jurídica existe una discusión acerca de si los principios morales pueden ser o no parte de los criterios de validez jurídica. Esto no tiene una respuesta pacífica. Así, por ejemplo, se ha desarrollado el debate entre positivistas incluyentes y excluyentes. Los primeros aceptan que entre los criterios de validez existan principios morales. Los segundos rechazan esta tesis (véase Leslie Green, Legal Positivism, en The Stanford Encyclopedia of Philosophy (E. Zalta ed., Stanford University, 2009). https://plato.stanford.edu/archives/fall2009/entries/legal-positivism

46 Carlos Nino, supra nota 7. 
lugar, existen límites jurídicos, o sea, los establecidos por el propio ordenamiento jurídico.

En cuanto a este tipo de límites las constituciones pueden ser flexibles o rígidas. Son rígidas aquellas constituciones que establezcan límites jurídicos a los poderes de reforma y flexibles las que no lo hagan ${ }^{47}$ Los límites jurídicos pueden ser: 1) materiales cuando se prohíbe la modificación (o incorporación) de determinados contenidos (por ejemplo, si se prohíbe la modificación de los derechos fundamentales); 2) formales cuando se contemplan exigencias orgánicas o procedimentales específicas para la validez de las reformas ${ }^{48}$ 3) lógicos o conceptuales son aquellos que no provienen del contenido de las disposiciones constitucionales, sino que se derivan de la estructura lógica del concepto de constitución adoptado. ${ }^{49}$ Un ejemplo específico de este tipo de límites es la imposibilidad de flexibilizar los procedimientos de reforma dentro de una constitución rígida. Así, en tanto una condición necesaria de las constituciones rígidas es que los procedimientos de reforma sean más complejos que el del resto de normas jurídicas, entonces un límite conceptual al poder de

47 Las constituciones pueden ser rígidas o flexibles según se contemple o no un proceso de reforma distinto al de las leyes. Así, una constitución es rígida "en la medida en que su modificación solo es posible a través de un procedimiento más complejo que el procedimiento legislativo ordinario". Víctor Ferreres Comella, Una defensa de la rigidez constitucional, 23 Doxa: Cuadernos de filosofia del derecho 29-47 (2000). http://www.cervantesvirtual.com/nd/ark:/59851/ bmc902g2. De esta manera, la rigidez de la Constitución implica que la validez de sus reformas se condiciona al cumplimiento de procedimientos que son, de alguna manera, más dificultosos que los necesarios para la validez del resto de normas del ordenamiento jurídico. La Constitución colombiana es rígida en tanto contempla procedimientos específicos y complejos para las reformas constitucionales.

48 Los límites formales dependen de la manera como se implementen dos variables: 1) el órgano de producción y 2) el procedimiento de reforma constitucional. La primera está en función de la posibilidad de diferenciar entre los órganos con poderes legislativos ordinarios y los órganos con poder de reforma constitucional. La segunda se refiere a que las reformas constitucionales deben hacerse mediante procedimientos más exigentes y complejos que los necesarios para crear normas infraconstitucionales. Debe tenerse en cuenta que, aun cuando es cierto que una constitución es más rígida si da una respuesta afirmativa a las dos variables (órganos y procedimientos distintos), también lo es que solo la segunda sea una condición necesaria de la rigidez). P. Pactet y F. Melin-Soucramanien, Derecho constitucional, Duque, C. (Trad.) 66 (Legis, Bogotá, 2011). Víctor Ferreres Comella supra nota 47. Valbuena Cisneros, Arminda, Límites y control a la reforma constitucional en México, Díkaion, 16, 157-178 (2007). http:// dikaion.unisabana.edu.co/index.php/dikaion/article/view/1380/1519

49 La tesis de los límites lógicos proviene del famoso artículo de Alf Ross, Sobre la autorreferencia y el dificil problema del derecho constitucional, en el concepto de validez y otros ensayos, Trad. E. Bulygin y E. Garzón (Ediciones Fontamara, 3ª. Ed., México, 1997). 
reforma consiste en modificar esos procedimientos en el sentido de hacerlos tan flexibles como las normas infraconstitucionales.

Una constitución es rígida cuando sus procedimientos de reforma son más difíciles de llevar a cabo. Es decir, cuando, por ejemplo, para reformar la constitución se exigen "mayorías más estrictas y procesos de aprobación más largos". ${ }^{50}$ La existencia de este tipo de constitución conlleva un límite conceptual o lógico: no es posible modificar los mecanismos de reforma hasta el punto de igualar o reducir las dificultades procedimentales al de las normas infraconstitucionales. En todo caso, en relación con la rigidez constitucional es necesario precisar dos asuntos. Primero, la rigidez implica dificultad para reformar, más no imposibilidad para hacerlo. Por esta razón, las cláusulas pétreas no son una manifestación necesaria de la rigidez constitucional. Segundo, de todas las normas jurídicas es posible predicar cierto grado de rigidez, como que se deben cumplir determinados requisitos para su modificación o derogación. Lo que en concreto exige la rigidez constitucional es que dentro de los distintos procedimientos de reforma, el de las normas constitucionales sea el más exigente.

En resumen, la rigidez constitucional es un límite jurídicoconceptual de los poderes de reforma y, en consecuencia, una condición de validez jurídica de las normas constitucionales creadas por los poderes de reforma. De acuerdo con esto, las constituciones rígidas incluyen de forma necesaria la siguiente regla conceptual implícita: "[s]i el órgano O mediante el procedimiento "P" dicta una disposición de reforma constitucional [LR], entonces [LR] es válida" ${ }^{51}$ Esta regla supone los siguientes elementos: 1) solo O puede dictar esas normas; 2) O no puede delegar esa competencia; 3) $\mathrm{O}$ solo puede hacer LR mediante "P" y 4) "P" debe ser más complejo que cualquier otro P para la creación de normas dentro del sistema. En este sentido, toda vez que la complejidad del procedimiento es una condición necesaria

50 Corte Constitucional, Sala Plena, Sentencia C-740-13, Expediente D-9552. Magistrado Ponente: Nilson Pinilla Pinilla.

51 Josep J. Moreso, Disposiciones de reforma constitucional. 202, Doxa 10, 201-222 (1991). https:// dialnet.unirioja.es/servlet/articulo?codigo $=128506$ 
de las constituciones rígidas, y un límite conceptual del poder de reforma, si un O modifica " $\mathrm{P}$ " haciéndolo tan flexible como cualquier o algún otro $\mathrm{P}$ entonces estaría violando la existencia misma de la constitución rígida; a saber, "que determinadas materias solo puedan ser reguladas por un determinado órgano y mediante determinado procedimiento". ${ }^{52}$ En palabras más simples, LR, en tanto es producto del poder de reforma, no es válida si establece que "P" es tan flexible como cualquier o algún otro $\mathrm{P}$ para la creación de normas infraconstitucionales. Como consecuencia de la rigidez constitucional es posible señalar la siguiente regla de validez implícita: LR es válida si y solo si es realizada por el órgano "O" competente por medio del procedimiento "P"; en donde "P" debe ser más complejo que cualquier otro $\mathrm{P}$ para la creación de normas dentro del sistema. Y en aplicación de esta regla conceptual implícita es necesario concluir que una reforma es inconstitucional si, por una parte, despoja de competencia a $\mathrm{O}$, y por la otra, existe algún procedimiento $\mathrm{P}$ que es al menos igual de complejo a "P".

En la Sentencia C-699 de $2016^{53}$ se trató específicamente el problema de si, al crearse un procedimiento de reforma constitucional que consta únicamente de una vuelta y cuatro debates, es incompatible con la rigidez constitucional. Para la Corte los FT no violan la rigidez constitucional toda vez que, en primer lugar, a pesar de que adiciona un mecanismo de reforma constitucional, este se inscribe en un contexto de transición, esto es, la terminación de un conflicto armado. En segundo lugar, es especial y transitorio, lo que significa que no se suprimen ni suspenden los otros mecanismos permanentes de enmienda constitucional. En tercer lugar, la rigidez de la Constitución se mantiene por encima de las leyes, pues se requiere mayoría absoluta.

Para desarrollar esta tesis, la Corte Constitucional argumenta que la rigidez significa que las normas constitucionales deben

52 Ibid., 203-204.

53 Corte Constitucional, Sala Plena, Sentencia C-699-2016, Expediente D-11601. Magistrado Ponente: María Victoria Calle. 
tener un "nivel específico de resistencia al cambio" que se caracteriza por cuatro elementos: 1) resistencia relativa, es decir que la resistencia al cambio de las normas constitucionales debe "ser superior a la de las leyes, pero el grado de superioridad es un aspecto susceptible de adaptación transicional",54 2) resistencia variable, toda vez que la inmodificabilidad de las cláusulas de reforma no forma parte de la identidad de la Constitución:55 3) resistencia diversa, en el sentido de que, para la Corte, una característica de la Constitución colombiana es la existencia de diferentes órganos y procedimientos dispuestos para la reforma de sus normas y 4) resistencia funcionalmente diferenciada. Para explicar este último elemento, la Corte recuerda su doctrina acerca de la diferencia entre reforma constitucional y sustitución. ${ }^{56}$ De acuerdo con esta doctrina, la Constitución se sustituye cuando se suprime, sustrae o reemplaza "uno de sus rasgos esenciales" a tal "punto que resulte incompatible con la identidad de la Constitución de 1991". A partir de estas consideraciones, la Corte concluyó que el principio de rigidez constitucional se caracteriza por que "sus normas son más resistentes al cambio que las de la ley y contemplan requisitos que promueven una mayor participación y consenso, resistencia susceptible de adaptarse a la transición" y, agrega la Corte que:

[1]os mecanismos de reforma de la Constitución pueden entonces ser modificados, en la medida en que no se sustituyan estos elementos por otros opuestos o integralmente diferentes. Pero el orden constitucional sería irreconocible si, por ejemplo, sus niveles de resistencia al cambio son idénticos a los de la ley pues deben ser superiores. ${ }^{57}$

54 Ibid.

55 Ibid

56 Ibid. Un estudio crítico del control constitucional material de las reformas desarrollado por la Corte Constitucional mediante la tesis de la sustitución puede verse en Vicente. F Benítez, Constitución popular, no judicial. Una teoría democrática del control de constitucionalidad de las reformas a la Constitución en Colombia (Temis-Universidad de La Sabana, Bogotá, 2014). Un estudio comparado de las diferentes estrategias de los tribunales constitucionales para desarrollar control material de las reformas Constitucionales véase Sabrina Ragone, El control material de las reformas constitucionales en perspectiva comparada. Teoria y realidad constitucional, 31 (2013), 391-405.

57 Corte Constitucional Sentencia, supra nota 52. 
La Corte Constitucional, por tanto, admite que la regla conceptual implícita de validez de los mecanismos de reforma explicada en el apartado anterior y, según la cual, es necesario que los procedimientos de reforma de las normas constitucionales ("P") deben ser más complejos que cualquier otro procedimiento de creación de normas $(\mathrm{P})$ dentro del sistema. Teniendo en cuenta lo anterior, los FT - a diferencia de lo que concluye la Corte Constitucional-sí sustituyen la Constitución: si uno de sus principios es que sea per se la norma más difícil de modificar del sistema, la existencia de los FT es incompatible con el principio de rigidez constitucional, pues reduce la complejidad del procedimiento para efectuar reformas por esa vía al de algunas leyes. En efecto, las leyes estatutarias exigen cuatro debates, mayoría absoluta, trámite en una legislatura y control automático de constitucionalidad (esto es, indudablemente, un procedimiento igual de complejo al de los FT). Se reitera, por tanto, que la rigidez constitucional implica que una reforma constitucional es válida si y solo si: 1) es realizada por el órgano "O" competente y 2) por medio del procedimiento "P" que debe ser más complejo que cualquier otro $\mathrm{P}$ para la creación de normas dentro del sistema. Puede estar abierta a la discusión si AL despojó al Congreso de la competencia de reforma (al eliminarse la iniciativa normativa). Pero no existe ninguna duda acerca de que el procedimiento definido en AL para la aprobación de los FT es inconstitucional pues existen otros $\mathrm{P}$ que son igual o más rígidos que los del FT (como las leyes estatutarias y orgánicas, etc.). 


\section{CONCLUSIONES}

Una norma $\mathrm{N}$ es válida si y solo si existe una $\mathrm{N} 2$ que autoriza a $\mathrm{O}$, mediante $\mathrm{P}$, para que expida $\mathrm{N} 1$ con el contenido $\mathrm{C}$. Una norma NA es aplicable jurídicamente si y solo si existe una norma N2 válida que establezca que N1 "debe" ser aplicada por los operadores del sistema. Por último, una norma NF tiene fuerza vinculante siempre que, de acuerdo con las consideraciones morales existentes, se exija su cumplimiento o seguimiento. Como se anotó, el hecho de que una norma pertenezca a un ordenamiento jurídico (que cumpla con las condiciones de validez) no significa que necesariamente tenga fuerza vinculante. Pero también se observó que el derecho pretende que la validez de las normas cumpla un rol normativo relevante. Para ello es necesario que las condiciones de validez de las normas tengan un fundamento moral.

En particular, el derecho pretende vincular, con independencia de lo que los individuos en general y los operadores jurídicos en particular evalúen caso a caso, la adecuación moral de cada una de las normas. Si es necesario, por ejemplo, que los operadores jurídicos tengan que evaluar de forma exhaustiva la aplicabilidad moral de todas las normas, entonces el derecho dejaría de tener relevancia práctica. Para que el derecho vincule, en suma, es necesario que tenga autoridad moral. ${ }^{58}$ Desde luego que en los casos en los que el derecho tiene autoridad moral las normas vinculan siempre que sean creadas de acuerdo con las condiciones de validez existentes. Si, por ejemplo, una norma es creada por un órgano incompetente o mediante un procedimiento incorrecto entonces no pueden pretender vincular, en tanto normas jurídicas, a los individuos y operadores jurídicos.

Este problema abre la discusión acerca de la situación jurídica de las normas que son creadas sin el cumplimiento de las

58 Joseph Raz, The Problem of Authority: Revisiting the Service Conception, Minnesota Law Review, 90, 1003-1044 (2006). http://www.minnesotalawreview.org/wp-content/uploads/2011/11/ Raz_3fmt.pdf. John Finnis, Natural law and natural rights ( $2^{\mathrm{a}}$ ed., Oxford University Press, Oxford, 2011). 
condiciones de validez. Este es el caso de los FT en el derecho constitucional colombiano. En los apartados anteriores se mostró que son condiciones de validez de los mecanismos de reforma constitucional que sus procedimientos sean democráticos y rígidos. Se concluyó, en primer lugar, que la restricción en las iniciativas normativas, la reducción en número y calidad de los debates y la obligación de aprobar los proyectos mediante votaciones en bloque y en corto tiempo, reduce sustancialmente la capacidad del procedimiento para lograr resultados legítimos e imparciales de la forma como lo exige el principio democrático. Y, en segundo lugar, se demostró que el procedimiento definido en AL para la aprobación de los FT es inválido en tanto se incumple la regla según la cual es necesario que el procedimiento "P", mediante el cual se pretenden crear normas constitucionales, sea más complejo que cualquier otro $\mathrm{P}$ para la creación de normas dentro del sistema.

Siendo los FT normas inválidas dentro del sistema jurídico colombiano, no es posible que pretendan autoridad jurídica (dejando nota, por cierto, que si fueran válidos tendrían la capacidad de vincular siempre y cuando el derecho colombiano cuente con autoridad moral). Esto es así toda vez que los FT, en tanto disposiciones que no reúnen las condiciones de pertenencia al sistema jurídico colombiano, no pueden pretender autoridad jurídica respecto de los individuos y operadores jurídicos. Algunos pueden pensar equivocadamente que, toda vez que la Corte Constitucional declaró la validez del AL (que es, a su vez, origen de los FT) entonces los FT son válidos. Sin embargo, debe recordarse que, como se advirtió antes, el hecho de que un juez (aun cuando sea un órgano de cierre como la Corte Constitucional) considere que una norma es válida o aplicable no significa que en verdad lo sea. En estos casos si una norma es inválida no puede, desde luego, pretender autoridad jurídica. Es decir, no puede pretender que vincule a los individuos con independencia de la evaluación moral del contenido de las normas.

En este contexto, las normas creadas en los FT serían aplicables solo en el caso de que existiera una norma jurídica vá- 
lida (perteneciente al derecho colombiano) que estableciera el "deber" de aplicar normas inválidas. Con algo de imaginación podríamos recurrir, por ejemplo, al artículo 241 de la Constitución que le otorga carácter definitivo a las decisiones de la Corte Constitucional. Se podría interpretar (nuevamente con alto grado de imaginación) que, a pesar de que en una sentencia de la Corte Constitucional se declaren válidas normas que en realidad son inválidas (en este caso inconstitucionales), en virtud de la competencia de órgano de cierre que le ha dado la Constitución se deben considerar sus decisiones aplicables. Esta tesis presenta diferentes problemas que la hacen inviable. Primero, no es posible afirmar que un error judicial pueda ser argumento suficiente para admitir la aplicabilidad de otras normas. Segundo, la declaración de constitucionalidad (errada) de la Corte Constitucional se refiere a AL y no respecto de cada uno de los eventuales FT que el Congreso llegue a expedir. Por último, lo que se afirma al respecto es si los FT son jurídicamente aplicables, es decir, si existe un "deber" entrecomillado de aplicarlos (esto es, en otras palabras, la descripción de un "deber" de aplicar los FT y no la identificación de un deber genuino de hacerlo). Para que exista el deber genuino debe mostrarse, al igual que en el caso de la validez como pertenencia, que existen criterios morales que justifican ese deber.

Nos queda, por tanto, una posibilidad: que los FT sean moralmente aplicables. Para ello es posible explorar dos caminos: primero, que el contenido de cada una de las normas creadas en los FT tenga contenido moral. Sin embargo, estas posibilidades se enfrentan a un problema mayor: no es posible realizar una tesis general acerca de la aplicabilidad de los FT pues dependerá de cada norma y de cada caso para que los jueces puedan definir si están o no conformes con las exigencias morales. Estos caminos nos llevan a una situación paradójica representada por dos opciones excluyentes: 1) las normas creadas en los FT son vinculantes solo si coinciden con las exigencias morales que son previas e independientes de ellos, caso en el cual los FT son irrelevantes en relación con las exigencias morales y por tanto 
superfluas respecto de lo que deben hacer los individuos o como deben decidir los operadores; o 2) los FT establecen normas distintas e independientes de las exigencias morales, caso en el cual no pueden pretender ningún valor normativo en relación de cómo deben actuar los individuos o decidir los operadores.

El segundo camino consistiría en afirmar que la finalidad de la paz justifica la aplicabilidad de los FT. Es decir, que (y argumentando en un sentido contrafáctico) si no existieran los mecanismos de reforma el orden social y los derechos de las personas se verían perturbados o, al revés: que sin esos mecanismos no sería posible lograr el orden ni la garantía de los derechos de las personas. Este camino, sin embargo, sigue siendo insatisfactorio. En primer lugar porque se basa en premisas empíricas que no son de ninguna manera evidentes: qué tanto servirán los FT para asegurar o recomponer el orden social es una hipótesis empírica que depende de distintas variables sociales y que, desde luego, nadie puede sostener sin tener ningún tipo de duda. En segundo lugar, las condiciones de validez que los FT desconocen (como el carácter democrático de las reformas y la rigidez constitucional) tienen propósitos similares, a saber, conservar el orden social y proteger los derechos de las personas. En tercer lugar, algunos pueden sostener que los FT son un mecanismo para otorgar seguridad jurídica al Acuerdo como una condición indispensable para lograr los propósitos del proceso de paz en Colombia. Sin embargo, esa posición incurre claramente en una contradicción performativa. Estas contradicciones ocurren cuando el significado de un enunciado contradice los presupuestos de formular ese enunciado (piénsense en el reiterado ejemplo del enunciado "yo estoy muerto"). Así, se incurre en una contradicción performativa cuando se afirma que se "pretende otorgar seguridad jurídica de los acuerdos" mediante "la flexibilización de las normas que los implementan". 


\section{BIBLIOGRAFÍA}

\section{Libros}

Benítez, Vicente. F, Constitución popular, no judicial. Una teoría democrática del control de constitucionalidad de las reformas a la Constitución en Colombia (Temis-Uiversidad de La Sabana, Bogotá, 2014).

Estlund, David, La autoridad democrática. Los fundamentos de las decisiones políticas legitimas, trad. Sara Palacio Gaviria y Sebastián Linares (Siglo XXI Editores, Buenos Aires, 2011).

Finnis, John, Natural law and natural rights ( $2^{\mathrm{a}}$ ed., Oxford University Press, Oxford, 2011).

Gargarella, Roberto y Courtis, Christian, El nuevo constitucionalismo latinoamericano: promesas e interrogantes 10 (Santiago de Chile, Cepal, 2009). http://repositorio. cepal.org/bitstream/handle/11362/6162/1/S0900774_es.pdf

Habermas, Jürgen, Facticidad y validez (1 ${ }^{a}$. ed. Trotta: Madrid, 1998).

Hart, H. L. A., The concept of law, 176-177 (2nd ed. Ed. Penelope A. Bulloch and Joseph Raz, Clarendon Press, Oxford, 1994).

Kelsen, Hans, Teoría general del derecho y del Estado, trad. E. García Máynez, E. (5a reimpresión de la 2a ed., Universidad Nacional Autónoma de México, México, 1995).

Kelsen, Hans, Teoría pura del derecho, trad. R. Vernengo (14 ed., Editorial Porrúa, México, 2005).

Lancheros, Juan C., Mantilla, María, Pulido, Fabio, Suárez, Ingrid y Rincón, Rocío, Hoja de ruta. Trámite legislativo ordinario (Konrad Adenauer Stiftung, Bogotá, 2010).

Navarro, Pablo y Rodríguez, Jorge, Deontic Logic and Legal Systems (Cambridge University Press, New York, 2014).

Nino, Carlos Santiago, La validez del derecho (Editorial Astrea, Buenos Aires, 1986).

Nino, Carlos Santiago, La constitución de la democracia deliberativa, traducción Roberto P. Saba (Gedisa, Barcelona, 2003).

Nino, Carlos Santiago, El constructivismo ético (Centro de Estudios Constitucionales, Madrid, 1989).

Palacios Torres, Alfonso, Concepto y control del procedimiento legislativo (1 $1^{\mathrm{a}}$. ed., Universidad Externado de Colombia, Bogotá, 2005).

Pactet, Pierre y Ferdinand, Melin-Soucramanien, Derecho constitucional, Duque, C., Trad. (Legis, Bogotá, 2011)

Raz, Joseph, The Authority of Law. Essays on Law and Morality (Oxford University Press, Oxford, 1979).

Ross, Alf, El concepto de validez y otros ensayos, trad. E. Bulygin y E. Garzón (Ediciones Fontamara, $3^{\text {a }}$. Ed. México, 1997).

Tierney, Stephen, Constitutional Referendums. The Theory and Practice of Republican Deliberation (Oxford: Oxford University Press, 2012). 


\section{Contribución en obras colectivas}

Bulygin, Eugenio, Tiempo y validez, en Análisis lógico y derecho, 193-214 (C. Alchourron y E. Bulygin, (Eds.) Centro de Estudios Constitucionales, Madrid, 1991).

Green, Leslie, Legal Positivism, en The Stanford Encyclopedia of Philosophy (E. Zalta ed., Stanford University, 2009). https://plato.stanford.edu/archives/fall2009/entries/ legal-positivism

Pulido Ortiz, Fabio y Bustos, Geraldine, Momentos constitucionales y legitimidad de los procedimientos de reforma, en Procesos legislativos y ordenamiento constitucional: abordajes teóricos, 92-105 (ed. G. Eljach, CAEL, Bogotá, 2016).

Spector, Horacio, Democracia y control constitucional: una tensión aparente, en Homenaje a Carlos Nino, 231-247 (M. Alegre, et. al., La Ley, Buenos Aires, 2008).

Waluchow, Will, Four Concepts of Validity: Reflections on Inclusive and Exclusive Positivism, en The Rule of Recognition and the United States Constitution, 123144 (en M. Adler and K. Himma (Eds.) Oxford University Press, Oxford, 2009).

\section{Revistas}

Cianciardo, Juan, La paradoja de la irrelevancia moral del gobierno y del Derecho. Una aproximación desde el pensamiento de Carlos Nino, 19 Díkaion, 47-70 (2010) b. http://dikaion.unisabana.edu.co/index.php/dikaion/article/view/1702

Del Rosario-Rodríguez, Marcos Francisco, La supremacía constitucional: naturaleza y alcances, 20 Díkaion 97-117 (2011). http://dikaion.unisabana.edu.co/index.php/ dikaion/article/view/1950/2506

Ferreres Comella, Víctor, Una defensa de la rigidez constitucional, 23 Doxa: Cuadernos de filosofía del derecho 29-47 (2000). http://www.cervantesvirtual.com/nd/ ark:/59851/bmc902g2

García Jaramillo, Leonardo, Recepción de postulados deliberativistas en la jurisprudencia constitucional. Análisis crítico a partir del tribunal colombiano, 10, Revista Argentina de Teoría Jurídica (2008). http://www.utdt.edu//ver_contenido. php?id_contenido $=2450 \& i d$ item_menu $=3555$

García Valverde, Facundo, Agencia política y legitimidad en la democracia deliberativa, 22, Eidos (2015), 225-252. http://www.scielo.org.co/scielo.php?script=sci_arttext \&pid=S1692-88572015000100011

Gargarella, Roberto, Pensar sobre la democracia, discutir sobre los derechos, 267, Nueva Sociedad (2017): 101-113.

González Quintero, Rodrigo, Procesos constituyentes y cortes constitucionales: una perspectiva comparada, 18 Díkaion, 135-161 (2009). http://dikaion.unisabana.edu. co/index.php/dikaion/article/view/1546/1868

Koh, Harold Hongju, The Fast Track and United States Trade Policy, 18 Brooklyn Journal of International Law, 1, 143-180 (1992).

Kristan, Andrej, A paradox of Hart's Fallible Finality, 2016, Analisi e diritto, 339-344 (2016). 
Moreso, Josep J., Disposiciones de reforma constitucional. Doxa 10, 201-222 (1991), 202. https://dialnet.unirioja.es/servlet/articulo?codigo $=128506$

Raz, Joseph, Reasoning with rules, 54, Current Legal Problems, 1-18 (2001).

Raz, Joseph, The Problem of Authority: Revisiting the Service Conception. Minnesota Law Review, 90, 1003-1044 (2006). http://www.minnesotalawreview.org/wpcontent/uploads/2011/11/Raz_3fmt.pdf

Ragone, Sabrina. El control material de las reformas constitucionales en perspectiva comparada. Teoría y realidad constitucional, 31 (2013), 391-405.

Tole, José Julián, Los TLC de Estados Unidos con países latinoamericanos: un modelo de integración económica "superficial" para el continente americano. Revista Derecho del Estado (30), 251-300. http://revistas.uexternado.edu.co/index.php/ derest/article/view/3525

Valbuena Cisneros, Arminda, Límites y control a la reforma constitucional en México, Dikaion, 16, 157-178 (2007). http://dikaion.unisabana.edu.co/index.php/dikaion/ article/view/1380/1519

\section{Jurisprudencia y normatividad colombiana}

Colombia, Constitución Política, versión corregida, 116 Gaceta Constitucional, 20 de julio de 1991. http://www.secretariasenado.gov.co/senado/basedoc/constitucion_politica_1991_pr012.html

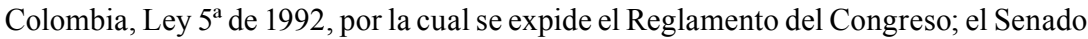
y la Cámara de Representantes, 40483 Diario Oficial, 18 de junio de 1992. http:// www.secretariasenado.gov.co/index.php/ley-5-de-1992

Colombia, Acto Legislativo 1 de 2016, por medio del cual se establecen instrumentos jurídicos para facilitar y asegurar la implementación y el desarrollo normativo del acuerdo final para la terminación del conflicto y la construcción de una paz estable y duradera, 49927 Diario Oficial, 7 de julio de 2016. http://www.secretariasenado. gov.co/senado/basedoc/acto_legislativo_01_2016.html

Corte Constitucional sentencias C-180 de 1994, Sala Plena, Expediente PE-005. Magistrado Ponente: Hernando Herrera Vergara.

Corte Constitucional, Sala Plena, Sentencia C-222 de 1997, Expediente D-1465. Magistrado Ponente: José Gregorio Hernández.

Corte Constitucional Sentencia C-387-97, Sala Plena, Expediente D-1545. Magistrado Ponente: Fabio Morón Díaz.

Corte Constitucional, Sala Plena, Sentencia C-1707 de 2000, Expediente OP-037. Magistrado Ponente: Cristina Pardo Schlesinger.

Corte Constitucional Sentencia C-140 de 2010, Sala Plena, Expediente D-3075. Magistrado Ponente: Humberto Sierra Porto.

Corte Constitucional, Sentencia C-150 de 2015, Sala Plena, Expediente PE-038. Magistrado Ponente: Mauricio González Cuervo.

Corte Constitucional, Sala Plena, Sentencia C-699 de 2016, Expediente D-11601. Magistrado Ponente: María Victoria Calle. 
